\title{
Heterotopic mesenteric ossification: Report of two cases with review of the literature
}

\author{
John Mussatto ${ }^{1}$, Stephen Albrecht ${ }^{1}$, Eric Ebaugh², Kahdi Udobi² and Ossama Tawfik ${ }^{1, *}$ \\ 1 Departments of Pathology and Laboratory Medicine, The University of Kansas Medical Center, Kansas City, Kansas, USA \\ 2 Trauma and Critical Care, The University of Kansas Medical Center, Kansas City, Kansas, USA
}

\begin{abstract}
Very few cases of heterotopic mesenteric ossification (HMO) have been reported in the literature. We describe two cases, one at an early phase and the other at a more advanced stage of the disease. Both patients developed HMO after multiple major abdominal surgeries. The value of monitoring serum alkaline phosphatase and calcium in a setting of previous abdominal surgery or trauma along with other clinical, radiological and pathologic findings is discussed.
\end{abstract}

Keywords: heterotopic mesenteric ossification; HMO; abdominal pain

\section{Introduction}

Heterotopic ossification is a rare benign condition defined as osteogenesis occurring in non-ossifying soft tissue sites $[1,2]$. While in itself uncommon, heterotopic ossification occurring intra-abdominally is extremely rare [3-8]. Heterotopic ossification of the intestinal mesentery was first described in 1983 by Hansen et al., but it was not until 1999 that Wilson et al. detailed five cases of intra-abdominal heterotopic ossification occurring in the mesentery that they coined as heterotopic mesenteric ossification (HMO) for which we will use to describe our case [9, 10]. HMO has also been referred to as intra-abdominal heterotopic bone formation, intra-abdominal myositis ossificans, mesenteritis ossificans, and heterotopic ossification of the mesentery in the literature $[11,12]$. We report two additional cases of $\mathrm{HMO}$ at various stages of the disease process along with careful review of the literature. HMO is a difficult preoperative diagnosis to make; an elevated alkaline phosphatase in setting of previous extensive abdominal trauma or surgery should raise the suspicion of this rare condition. The clinical, radiological, laboratory and histopathologic changes are discussed.

\section{Case report \#1}

The first patient is 55-year-old man who presented to the emergency department with abdominal pain, fevers, and chills on 9/8/14. Four days prior to admission he had consumed a large amount of Pepto-Bismol for diarrhea only to later develop constipation and his previously mentioned symptoms on admission. Relevant medical and surgical history included left and right acoustic neuromas, neurofibromatosis, seizures, hypercholesterolemia, anemia, degenerative joint disease, spondylosis of lumbar joint, ventriculoperitoneal shunt and tonsillectomy.
Medications on admission included cholecalciferol, cyclobenzaprine (Flexeril), diphenhydramine (Benadryl), melatonin, naproxen, pantoprazole DR, tramadol, sodium phosphate and multivitamins.

The patient was admitted to the hospital for a diagnosis of diverticulitis and was started on intravenous (IV) antibiotics, hydration, and a bowel regimen. However, over the following few days his pain and abdominal distension continued to increase and a repeat computed tomography (CT) scan revealed increased abdominal fluid and free air. He was emergently taken to surgery on 9/12/14 where a perforated sigmoid diverticulitis and fecal peritonitis were diagnosed intraoperatively. He underwent a sigmoid colon resection and washout and a decision was made to bring him back in 48 hours for a second look operation. At the second operation on 9/14/14 a second lavage was performed and a colostomy was brought out and matured. Over the following weeks the patient's post-operative course was complicated by bilateral peripheral pulmonary emboli as well as the development of gastrointestinal

*Corresponding author: Ossama Tawfik, MD, PhD., Professor, Vice Chairman, Director of Anatomic and Surgical Pathology, Department of Pathology and Laboratory Medicine, The University of Kansas Medical Center, Kansas City, Kansas 66160, USA. Tel.: 913-588-1185; Fax: 913-5888780; Email: otawfik@kumc.edu

Received 4 December 2015 Revised 16 January 2015 Accepted 22 January 2015 Published 30 January 2016

Citation: Mussatto J, Albrecht S, Ebaugh E, Udobi K, Tawfik O. Heterotopic mesenteric ossification: Report of two cases with review of the literature. $J$ Mod Hum Pathol. 2016; 1(1):6-10. DOI:10.14312/2397-6845.2016-2

Copyright: (® 2016 Mussatto J, et al. Published by NobleResearch Publishers. This is an open-access article distributed under the terms of the Creative Commons Attribution License, which permits unrestricted use, distribution and reproduction in any medium, provided the original author and source are credited. 
bleeding while on therapeutic Lovenox. He ultimately had an inferior vena cava filter placed and his pulmonary emboli resolved. The patient's condition improved and he was discharged on 10/14/14.

In the following months the patient experienced no notable complications from his colectomy and colostomy. Following a normal colonoscopy a decision was made to undergo a colostomy reversal procedure. He was admitted on 9/14/15 for the colostomy reversal. Intraoperatively there were a considerable amount of dense adhesions found requiring adhesiolysis to free the underlying bowel and omentum. In the mesentery numerous bony plaques were encountered and deemed a risk for bowel perforation, and were thus excised (Figure 1). The rest of the bowel was inspected and found to be normal. The colostomy was detached, the colon was mobilized, and the anastomosis of the bowel was completed without complication. The patient's recovery was complicated by prolonged return of bowel function. Consequently a peripherally inserted central catheter line was placed on $9 / 25$ to provide total parenteral nutrition (TPN). In the subsequent days the patient was stabilized. His diet was slowly advanced, TPN was titrated down, and his bowel function returned. The patient was ultimately discharged on 10/11/15 in stable condition.

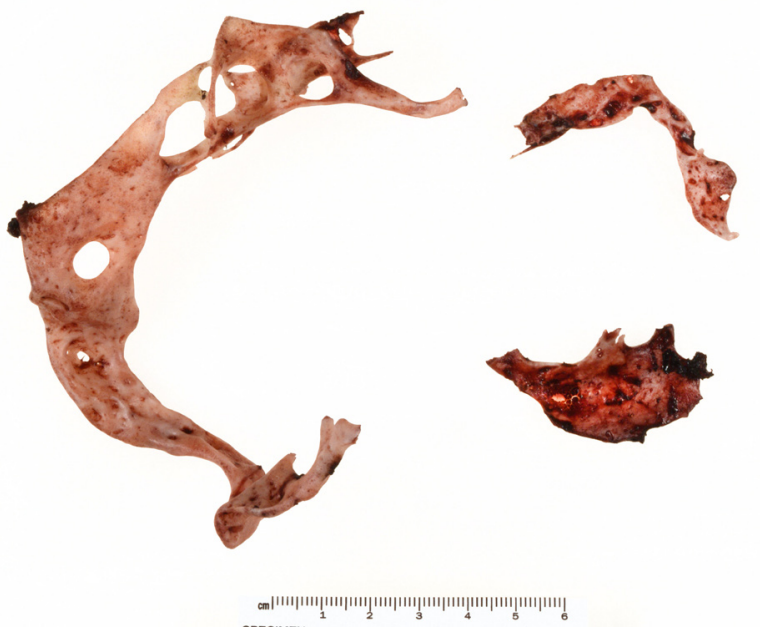

Figure 1 Excised well-formed bone fragments from the mesentery (heterotopic mesenteric ossification).

It is worth noting that upon chart review the patient's alkaline phosphatase (ALP) levels began to become elevated on 9/20/14 (157 U/L, normal 25-110 U/L), peaked on 10/2 (1654 U/L), and returned to within normal limits on 10/20 (Figure 2A). On the second admission, ALP levels became slowly started to increase above normal levels ranging between 119 to $155 \mathrm{U} / \mathrm{L}$ from 9/19/2015 to 9/30/2015. ALP level peaked at $339 \mathrm{U} / \mathrm{L}$, slowly dropping to $158 \mathrm{U} / \mathrm{L}$ on his discharge day, 10/11/2015 (Figure 2A). Serum calcium remained mainly within normal limits except with slight drop below normal to 7.9MG/DL (normal 9-11 MG/DL). That drop coincided with ALP elevation and with flare up of HMO disease activity (Figure 2B).

\section{Case report \#2}

A 60-year-old male, previously in excellent health, had presented with symptoms of fever, chills, fatigue, abdominal pain, nausea, vomiting, and diarrhea. At

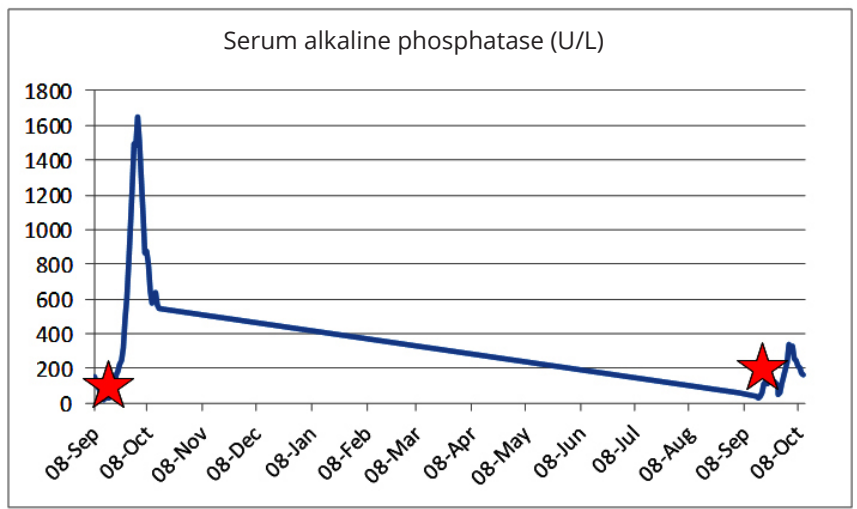

(A)

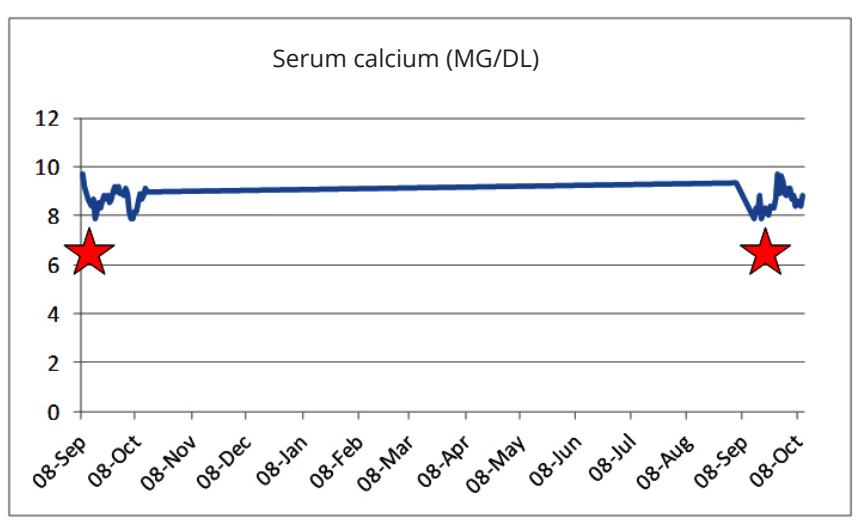

(B)

Figure 2 Graphs of serum alkaline phosphatase (ALP) in (A) and serum calcium in (B) showing alteration of serum levels corresponding with disease activity in patient \#1. The patient's alkaline phosphatase (ALP) levels began to become elevated on 9/20/14 (157 U/L, normal 25-110 U/L), peaked on 10/2 (1654 U/L), and returned to within normal limits on 10/20 (Figure 2A). On the second admission, ALP levels became slowly started to increase above normal levels ranging between 119 to $155 \mathrm{U} / \mathrm{L}$ from 9/19/2015 to 9/30/2015. ALP level peaked at $339 \mathrm{U} / \mathrm{L}$, slowly dropping to $158 \mathrm{U} / \mathrm{L}$ on his discharge day, 10/11/2015 (Figure 2A). Serum calcium remained mainly within normal limits except with slight drop below normal to 7.9MG/DL (normal 9-11 MG/DL) (Figure 2B).

first he met with his primary care physician (PCP) who prescribed him empiric ciprofloxacin and imodium. While on medication the patient started to have abdominal distention, as which point his PCP directed him to go to the Emergency Department urgently. There, at an outside hospital, he was found to have a possible small bowel obstruction. He was placed on nasogastric (NG) suction and had decompression. Imaging showed a persistent dilatation and his abdominal distension continued to worsen. An exploratory laparoscopy was then performed. However, during the procedure, the findings were unremarkable; the transition area of dilated bowel to normal bowel was not clearly evident, and no mechanical cause was discovered to explain the patient's obstruction. Following surgery, he was not passing flatus or having bowel movements. He was placed on an all-liquid diet and the NG tubed was discontinued. Days later he was taken back to surgery, after $\mathrm{CT}$ revealed a transition point, to have a re-exploration and small bowel resection with stapled and over-sewn anastomosis at the area where the caliber of small bowel transitioned from dilated to collapse. Again, during the procedure no mechanical cause could be identified. However, it was noted that the patient had cholelithiasis, but a cholecystectomy was not performed 
because of the increased morbidity to the patient at that time. Postoperatively, he did have a small amount of formed stool, but he continued to have abdominal distension, minimal abdominal pain, and nausea. He was then transferred to our facility for further evaluation and therapy.

On admission, the patient was instructed to withhold oral intake of flood and fluids and a NG tube was placed. Interestingly, he reported that when the NG tube was on low-intermittent suction, his symptoms of bloating/fullness and nausea/vomiting were relieved. He did report passing some gas and having small 'pasty' stools. It should be noted for completeness that his stool later came back positive for C. difficile, and Flagyl was started. Findings on CT showing multiple entero-enteric fistulas and mural thickening was concerning for Crohn's disease. However, the patient denied any previous symptoms of inflammatory bowel disease and a reported normal surgical pathology report from his outside hospital suggested otherwise. It was thought the patient had a partial small bowel obstruction at the previous surgery site likely due to post-operative edema. Because there was no indication for emergent surgery at the time, the hope was that the obstruction would resolve spontaneously with conservative treatment of NG tube decompression, TPN, IV fluids, and daily abdominal plain films to monitor his kidneys, ureters and bladder. After approximately three weeks following his previous surgery, the patient still had not made any significant improvement and imaging continued to show small bowel obstruction. It was determined that conservative management was not effective and he was taken into surgery for exploratory laparoscopy, lysis of adhesions and small bowel resection. During surgery, numerous dense adhesions were lysed. However, the area of obstruction was noted to be at the anastomosis site with multiple dense entero fistulas. The mesentery along this area (the proximal jejunum to the right colon) was significantly fibrotic and retracted. The area was so fibrotic that the surgeon was unable to adequately free the small bowel, and an extensive small bowel resection had to be preformed. The pathology report of the resected specimen came back as, "focal ectopic ossification of the mesentery ('mesenteritis ossificans') with mesenteric retraction" (Figure 3). Following the surgery, the patient had a full recovery. The last time he was seen was at his two-month post-op appointment in March 2011. During that time, he was doing well.

The patient's ALP levels were elevated at $131 \mathrm{U} / \mathrm{L}$ when admitted to our hospital on $01 / 14 / 2011$ and continued to increase and peaked at $250 \mathrm{U} / \mathrm{L}$ on $1 / 23 / 2011$. The ALP levels started to decline rapidly following surgery and returned to within normal limits for four days at $50 \mathrm{U} / \mathrm{L}$. Serum calcium levels were slightly low during the patient's stay ranging from 7.9 to 9.3 MG/DL and phosphorous levels remained normal for the nearly the entire time the patient was hospitalized. TPN effects could have contributed to his low calcium levels. The patient had slightly elevated AST/ ALT. It was suspected that it was due to cholestasis in the setting of prolonged NPO and TPN. At his two-month postop check-up AST/ALT, alkaline phosphatase, calcium and phosphorous were all within normal limits.

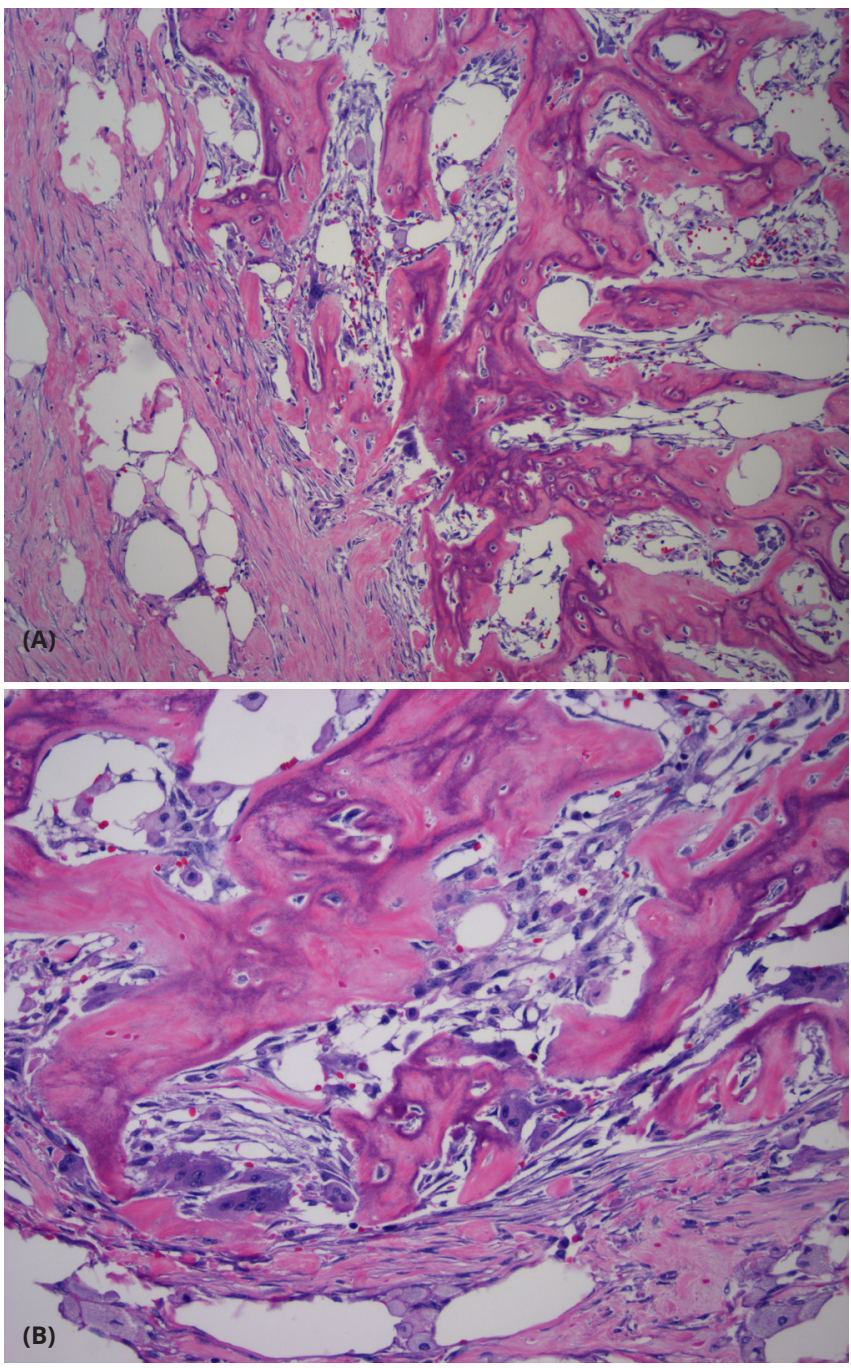

Figure 3 Photomicrographs of heterotopic mesenteric ossification showing the mesenteric tissue infiltrated by abundant heterotopic reactive woven bone trabeculae in a fibrous stroma with mild chronic inflammatory infiltrate, (Hematoxylin and eosin, magnification x100 and x200 in A and $B$, respectively).

\section{Discussion}

$\mathrm{HMO}$ is a condition that has rarely been reported in the literature. The majority of the patients reported with $\mathrm{HMO}$ have presented with a prior history of abdominal operations and/or intestinal obstruction but there are other HMO reported cases presenting with enterocutaneous fistulae, peritonitis, or incidentally found on imaging or intraoperatively $[11,12]$. The diagnosis of HMO preoperatively has been notably difficult. However, it is of clinical significance not only due to its potential complications such as small bowel obstruction, bowel perforation and persistent enterocutaneous fistula formation, but also because of its potential to be mistaken for a malignant process [11, 13-15].

Radiologically there may be several modalities of use to aid in the diagnosis. Arguably the best imaging tool for identifying $\mathrm{HMO}$ is $\mathrm{CT}$. When performed during the maturation process of $\mathrm{HMO}, \mathrm{CT}$ can also demonstrate progressive changes of these findings including identifiable cortical and trabecular architecture [16]. The features of $\mathrm{HMO}$ on CT have been observed as early as one month after 
the inciting event and include linear and branching highattenuation structures within the fat of the mesentery and omentum. Identifying $\mathrm{HMO}$ using CT can be challenging however, as the findings can be misidentified as contrast leakage, retained foreign body, ossifying neoplasm, dystrophic calcification, bowel ischemia, or active bleeding. To differentiate from these other possibilities a repeat CT scan should be obtained when in doubt. Bone formation remains static while contrast or blood changes in position and pool in dependent areas, and dystrophic calcification typically appears as faint radiodense areas that are punctate and irregular [11, 13, 15-17].

Other imaging modalities such as routine $x$-rays, radionuclide bone scan and Tc-99m MDP SPECT may also be of utility in the diagnosis. Heterotopic ossification may not show up on x-ray for up to 5 weeks if at all, but the demonstration of trabecular bone for a patient with HMO can be diagnostic [11, 13, 14, 16]. Radionuclide bone scan are sensitive but not specific for $\mathrm{HMO}$ and can show increased uptake in the course of HMO before becoming present on plain films [14]. Tc-99m MDP SPECT has shown intense osteoblastic activity inside an HMO lesion in one case, and CT scan with 3D reconstruction may also have diagnostic use $[15,6]$.

In general laboratory studies tend to be unremarkable. Very few reports suggested that an elevation in ALP levels may reflect an increase in osteoblastic activity and heterotopic bone formation. Serologically, elevated ALP levels have been evaluated in only five patients with $\mathrm{HMO}$ $[7,11,13,14,18]$. Recently Reynoso et al. has shown that serum ALP levels mirrored disease activity with peak levels reached approximately 3-4 weeks after an inciting event followed by normalization once HMO lesions were excised [11]. These investigators suggested that serum ALP could be a useful as sensitive but not specific diagnostic tool for diagnosing HMO. The elevation in ALP makes sense as it is normally elevated with osteoblastic activity and bone formation. In patients with heterotopic ossification in the extremities it has also been observed that these levels return to normal as the bone maturation process occurs [14]. It should also be noted that serum calcium and phosphorous levels have been found to be normal in all forms of heterotopic ossification and can help to exclude disorders of mineralization [14]. In both of our patients there was significant increase in ALP levels that coincided with disease activity. Of interest was the concomitant decrease in serum calcium. We were able to follow disease activity in one patient and demonstrated that each time the patient had significant elevation of ALP levels and reduction in serum calcium that coincided with flare up of the HMO condition (Figures $3 \mathrm{~A}$ and $\mathrm{B}$ ). In the less severe $\mathrm{HMO}$ condition in the other patient, the ALP levels were lower and clinically and radiologically there was no evidence of disease, suggesting a good correlation of ALP levels and disease intensity.

Classic histopathologic features of HMO include nodules of dense fibrous masses within the mesenteric tissue mostly composed of trabeculae of well-formed lamellar bone trabeculae with dystrophic calcification. The osteoblastic cells show usually no evidence of atypia, necrosis or increase in mitotic activity. HMO can sometimes exhibit a number of features that can elevate concerns for a malignant process including high cellularity, mitotic figures, and a "lace-like" osteoid production [17]. The most important histological feature of $\mathrm{HMO}$ is the zone formation. This refers to the progressive maturation from the central, inner immature zone consisting of hypercellular reactive fibrous tissue that can contain atypia and mitoses, to the middle zone of organized osteoid, to finally the peripheral zone of the lesion consisting of well-formed mature lamellar bone [11, $12,13,18]$. It is important to contrast this with sarcomas, which exhibit their most aggressive histopathologic changes at the periphery of the lesion [14].

The pathogenesis of HMO is currently unknown. It has been hypothesized that for HMO to occur four factors need to be present: an initial inciting event (e.g. trauma, sepsis, and surgery), the release of signals from injured or inflammatory cells, the presence of mesenchymal cells, and the genes of these mesenchymal cells to become activated by the signals released to cause differentiation into osteoblasts or chondroblasts [11]. Bone formation could occur from the seeding of particles containing osteogenic cells derived the perichondrium or periosteum of the pubic symphysis, sternum, or other bones that occur during trauma or surgery [13]. Research into the origin of $\mathrm{HMO}$ has focused on two genetic diseases that cause soft connective tissue to be replaced by mature heterotopic bone: fibrodysplasia ossificans progressiva and progressive osseous heteroplasia. As a result of investigating the molecular pathways involved in these two conditions much attention has been given to the role bone morphogenetic proteins (BMPs) and BMPs antagonists play in heterotopic bone formation and thus these molecules have also been implicated in the pathogenesis of HMO [14].

The treatment of HMO remains circumstantial. Surgical excision is recommended in symptomatic patients only, while careful observation is most often recommended for those who are asymptomatic and found to have $\mathrm{HMO}$ incidentally on imaging $[11,13]$. It has been suggested that nonsteroidal anti-inflammatory drugs may have some prophylactic benefit in preventing heterotopic ossification by inhibiting prostaglandin production. Prostaglandins are known co-stimulators of heterotopic bone formation along with the BMPs, although BMPs have been proven to still be able to act downstream of this inhibitory effect [11, 14]. Bisphosphonates and radiotherapy may be useful in the prevention of heterotopic ossification recurrence in the extremities, although radiotherapy has obvious limited utility in the abdomen [11, 13, 14].

\section{Conclusion}

In summary, the preoperatively diagnosis of $\mathrm{HMO}$ is difficult due to the limited number of reported cases in the literature. Although $\mathrm{HMO}$ is a benign condition, its major morbidity is bowel obstruction, bowel perforation and persistent enterocutaneous fistula formation. We described two such cases at the two ends of the spectrum of the disease. Both patients developed $\mathrm{HMO}$ after multiple major abdominal surgeries. We have demonstrated that monitoring serum ALP and calcium levels in a setting of 
previous abdominal surgery or trauma is potentially valuable for accurate diagnosis and for monitoring disease activity. Serum ALP levels mirrored disease activity with peak levels around inciting events and normalized with HMO.

\section{Conflicts of interest}

Authors declare no conflicts of interest.

\section{References}

[1] Subbarao JV, Garrison SJ. Heterotopic ossification: diagnosis and management, current concepts and controversies. J Spinal Cord Med. 1999; 22(4):273-283.

[2] Zychowicz ME. Pathophysiology of heterotopic ossification. Orthop Nurs. 2013; 32(3):173-177.

[3] Lemeshev Y, Lahr CJ, Denton J, Kent SP, Diethelm AG. Heterotopic bone formation associated with intestinal obstruction and small bowel resection. Ala J Med Sci. 1983; 20(3):314-317.

[4] Yannopoulos K, Katz S, Flesher L, Geller A, Berroya R. Mesenteric ossification. Am J Gastroenterol. 1992; 87(2):230-233.

[5] Patel RM, Weiss SW, Folpe AL. Heterotopic mesenteric ossification: a distinctive pseudosarcoma commonly associated with intestinal obstruction. Am J Surg Pathol. 2006; 30(1):119-122.

[6] Schiergens TS, Reichelt A, Thasler WE, Rentsch M. Abdominal Bone Formation. J Gastrointest Surg. 2015; 19(3):579-580.

[7] Bovo G, Romano F, Perego E, Franciosi C, Buffa R, et al. Heterotopic mesenteric ossification ("intraabdominal myositis ossificans"): a case report. Int J Surg Pathol. 2004; 12(4):407-409.

[8] Torgersen, Osmolak A, Bikhchandani J, Forse AR. Ectopic bone in the abdominal cavity: a surgical nightmare. J Gastrointest Surg. 2013; 17(9):1708-1711.

[9] Hansen O, Sim F, Marton PF, Grüner OP. Heterotopic ossification of the intestinal mesentery. Report of a case following intraabdominal surgery. Pathol Res Pact. 1983; 176(2-4):125-130.

[10] Wilson JD, Montague C], Salcuni P, Bordi C, Rosai J. Heterotopic mesenteric ossification ('intraabdominal myositis ossificans'). Am J Surg Pathol. 1999; 23(12):1464-1470.

[11] Reynoso J, Christensen D, Latifi R. Heterotopic mesenteric ossification as a cause of persistent enterocutaneous fistula: overview of the literature and addition of a new case. Eur Surg. 2011; 44(4):285-290.

[12] Athanazio DA, de Carvalho AL, Silva NO, Athanazio PR. Heterotopic intraabdominal ossification: report of a case and review of the literature. Jornal Brasileiro de Patologia e Medicina Laboratorial. 2009; 45(2):125-130

[13] Ioannidis O, Sekouli A, Paraskevas G, Kotronis A, Chatzopoulos S, et al. Intra-abdominal heterotopic ossification of the peritoneum following traumatic splenic rupture. J Res Med Sci. 2012; 17(1):92-95.

[14] Kaplan FS, Glaser DL, Hebela N, Shore EM. Heterotopic ossification. J Am Acad Ortho Surg. 2004; 12(2):116-125.

[15] Deryk S, Goethals L, Vanhove C, Geers C, Vandenbroucke F, et al. Imaging Characteristics of Heterotopic Mesenteric Ossification on FDG PET and Tc-99m Bone SPECT. Clin Nucl Med. 2008; 33(7):496-499.

[16] Baker JC, Menias CO, Bhalla S. Bone in the belly: traumatic heterotopic mesenteric ossification. Emerg Radiol. 2012; 19(5):429-436.

[17] Binesh F, Akhavan A, Navabii H, Ostadi M. Heterotopic mesenteric ossification: report of a case and review of the literature. BMJ Case Rep. 2012; pii: bcr0220125793.

[18] Hashash JG, Zakhary L, Aoun EG, Refaat M. Heterotopic mesenteric ossification. Colorectal Dis. 2012; 14(1):e29-e30. 